\title{
Terbinafine Induced Liver Injury: A Case Report
}

\author{
Narendra S. Choudhary ${ }^{\star}$, Hardik Kotecha ${ }^{\dagger}$, Neeraj Saraf ${ }^{\star}$, Dheeraj Gautam${ }^{\ddagger}$, Sanjiv Saigal ${ }^{\star}$ \\ *Institute of Liver Transplantation and Regenerative Medicine, ${ }^{\dagger}$ Institute of Digestive and Hepatobiliary Sciences, and ${ }^{\ddagger}$ Institute of Histopathology, \\ Medanta, The Medicity, Sector 38, Gurgaon, Haryana, India
}

$\Lambda$ 55-year-old male presented to us with history of progressive jaundice and pruritus. He had been on Terbinafine $250 \mathrm{mg}$ once a day for onychomycosis for a total duration of 1 month after which he developed jaundice. He had no history of any systemic disease. Terbinafine was stopped and he was investigated thoroughly; his liver functions tests revealed mixed cholestatic hepatitis picture (bilirubin $13.6 \mathrm{mg} / \mathrm{dL}$ (direct fraction $11.7 \mathrm{mg} / \mathrm{dL}$ ), AST 119 U/L, ALT 216 U/L, GGT 205 U/L, ALP 202 U/L, $R$ ratio 3), blood counts were normal (hemoglobin $13.6 \mathrm{~g} / \mathrm{dL}$, total leukocyte count $9000 / \mu \mathrm{L}$, platelet count $160,000 / \mu \mathrm{L}$ ), viral serologies (IgM anti-HAV, IgM anti-HEV, HBsAg, anti-HCV, hepatitis B virus DNA, hepatitis C virus RNA) were negative, markers for autoimmune liver disease were negative (anti-nuclear antibody, anti-smooth muscle antibody, anti-mitochondrial antibody, anti-liver kidney microsomal antibody), he had normal values of serum Ceruloplasmin and Ferritin, USG abdomen and MRCP were negative for biliary obstruction. To further substantiate the diagnosis of drug induced hepatotoxicity and to exclude other causes, a liver biopsy was done which showed maintained lobular architecture with extensive intraductal and canalicular cholestasis, minimal lobular inflammation and portal tract expansion with mixed infiltrate along with extensive Kupffer cell hyperplasia; there was no suggestion of autoimmune hepatitis or granuloma formation (shown in Figure 1). A diagnosis of Terbinafine induced liver injury was made (RUCAM score 9, highly probable) and he was given Ursodeoxycholic acid $300 \mathrm{mg}$ thrice a day till normalization of liver function tests, which was for a total dura-

Keywords: Terbinafine, drug induced liver injury, liver

Received: 12.1.2014; Accepted: 3.3.2014; Available online: 3.4.2014

Address for correspondence: Sanjiv Saigal, Institute of Liver Transplantation and Regenerative Medicine, Medanta, The Medicity, Sector 38, Gurgaon, Haryana, India. Tel.: +919811552928

E-mail: sanjivsaigal@hotmail.com

Abbreviations: DILI: drug induced liver injury; ULN: upper limit of normal http://dx.doi.org/10.1016/j.jceh.2014.03.040 tion of three months. His liver function tests improved gradually during this period as shown in Figures 2 and 3.

\section{DISCUSSION}

DILI is rare with most of the drugs used; diagnosis is important as drug discontinuation is needed and it requires a high index of clinical suspicion. DILI has a wide spectrum of manifestations that include acute hepatitis, cholestatic hepatitis (with and without bile duct injury), steatosis/steatohepatitis, chronic hepatitis, granulomatous hepatitis, vascular lesions and tumors. ${ }^{1}$ Drug induced liver injury are difficult to diagnose as characteristic features are lacking, however, mixed hepatitis and cholestatic picture and features of hypersensitivity may suggest DILI. ${ }^{1}$ Several criteria are used to define DILI and RUCAM criteria is the most widely used. ${ }^{2,3}$ DILI is defined as serum alanine transaminase $\geq 5$ times elevation of upper limit of normal (ULN) or alkaline phosphatase elevation $\geq$ two times ULN or alanine transaminase $\geq 3$ ULN with elevation of bilirubin to $\geq 2$ ULN. Hepatitic or cholestatic types of liver

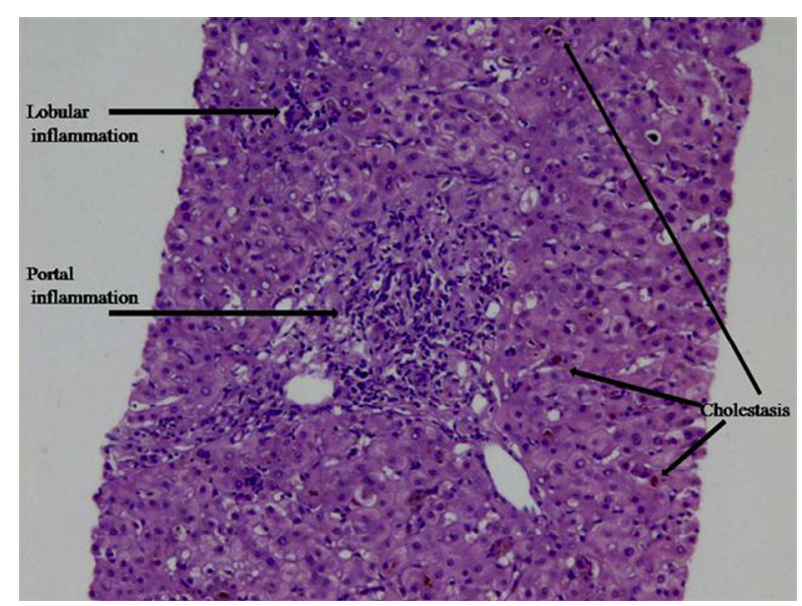

Figure 1 Liver biopsy showing cholestasis, portal and lobular inflammation. 


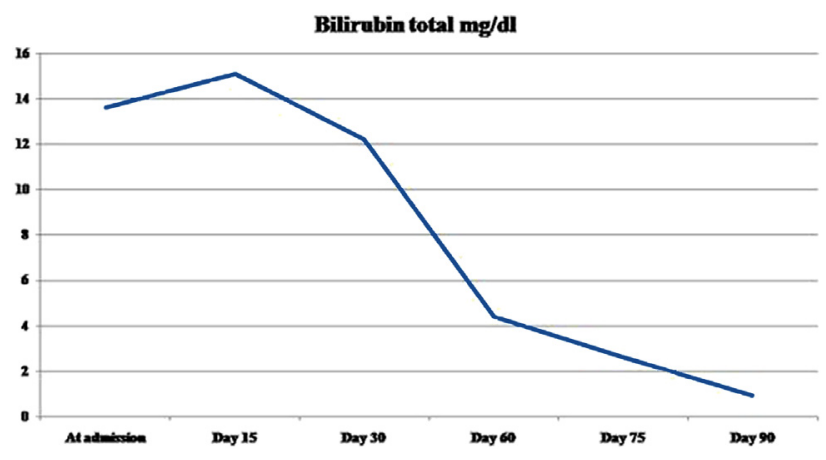

Figure 2 Dynamic changes in serum bilirubin levels over time.

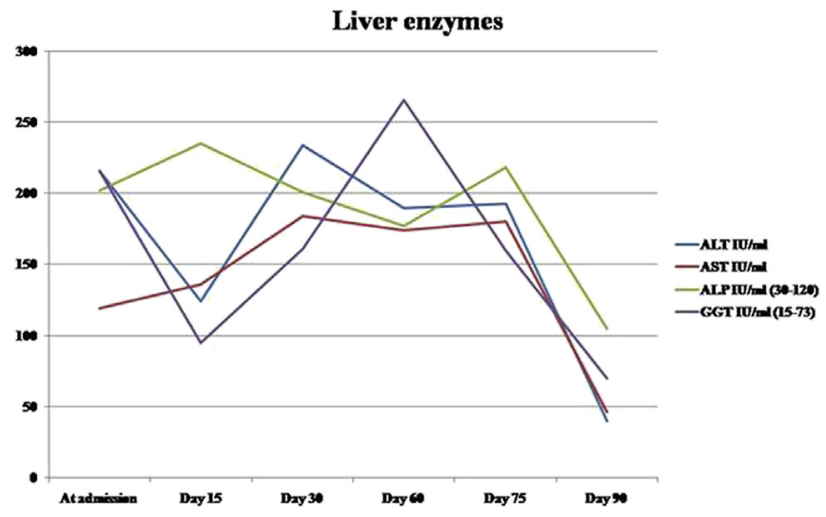

Figure 3 Dynamic changes in serum liver enzymes levels over time.

injury are defined by using $R$ value $(R=$ ALT ULN/ALP ULN); $R \geq 5$ is suggestive of hepatocellular injury while $R<2$ is suggestive of cholestatic injury and $R$ value in between 2 and 5 is suggestive of mixed type of injury. ${ }^{4}$ Terbinafine is a common antifungal drug used for onychomycosis that may cause hepatotoxicity with an incidence of 0.5-3/100,000 exposed. ${ }^{1}$ Terbinafine cause several types of hepatotoxicity that include acute hepatitis, cholestasis or mixed picture, acute liver failure and vanishing bile duct syndrome. ${ }^{1,5,6}$ Latency period between drug exposure and onset of jaundice is generally 2-6 weeks, serum bilirubin values may peak upto $3-5$ weeks after Terbinafine discontinuation and normalization of liver function tests may take 2-12 months. ${ }^{6,7}$ Most of the cases recover after discontinuation of the drug, however, rarely Terbinafine may cause acute or subacute liver failure requiring liver transplantation. ${ }^{8}$ Possible mechanisms of Terbinafine related liver injury include reaction to allylic aldehyde metabolite that may bind to hepatobiliary proteins to cause direct toxicity or by causing immune-mediated reaction to canalicular proteins leading to cholestasis. ${ }^{9}$ The histopathologic examination of liver biopsies generally show a mixed picture of hepatitis and cholestasis. ${ }^{7}$ There is no specific treatment, although Ursodeoxycholic acid is reported to help in recovery as seen in our case, ${ }^{10}$ and periodic monitoring of liver function tests should be done. ${ }^{5}$ Thus a high index of clinical suspicion supported by a liver biopsy helped us to diagnose Terbinafine induced mixed hepatitis-cholestatic liver injury.

\section{CONFLICTS OF INTEREST}

All authors have none to declare.

\section{REFERENCES}

1. Chitturi S, Farrell GC. Drug-induced liver disease. In: Schiff ER, Sorrell MF, Maddrey WC, eds. Schiff's Diseases of the Liver. 10th ed. Philadelphia: Lippincott Williams and Wilkins; 2007:924-1005.

2. Tajiri K, Shimizu Y. Practical guidelines for diagnosis and early management of drug-induced liver injury. World J Gastroenterol. 2008;14:6774-6785.

3. Shapiro MA, Lewis JH. Causality assessment of drug-induced hepatotoxicity: promises and pitfalls. Clin Liver Dis. 2007;11: 477-505.

4. Aithal GP, Watkins PB, Andrade RJ, et al. Case definition and phenotype standardization in drug-induced liver injury. Clin Pharmacol Ther. 2011;89:806-815.

5. Gupta AK, del Rosso JQ, Lynde CW, Brown GH, Shear NH. Hepatitis associated with terbinafine therapy: three case reports and a review of the literature. Clin Exp Dermatol. 1998;23:64-67.

6. Anania FA, Rabin L. Terbinafine hepatotoxicity resulting in chronic biliary ductopenia and portalfibrosis. Am J Med. 2002; 112:741-742.

7. Topalak O, Uçmak F, Sağol O, Akpinar H, Gönen O. Terbinafine induced prolonged cholestasis: case report and review of the literature. Turk J Gastroenterol. 2002;13:180-182.

8. Perveze Z, Johnson MW, Rubin RA, et al. Terbinafine-induced hepatic failure requiring liver transplantation. Liver Transpl. 2007;13: 162-164.

9. Iverson SL, Uetrecht JP. Identification of a reactive metabolite of terbinafine: insights into terbinafine-induced hepatotoxicity. Chem Res Toxicol. 2001;14:175-181.

10. Agca E, Akcay A, Simsek H. Ursodeoxycholic acid for terbinafineinduced toxic hepatitis. Ann Pharmacother. 2004;38:1088-1089. 